\title{
Unraveling the genetic causes in large pedigrees with gout by whole-exome sequencing
}

\author{
XIAORU XIA ${ }^{1 *}$, JING JIN $^{2 *}$, ZHEN-JI CHEN $^{3}$, ZHENNI ZHOU $^{4}$, HUI CHEN ${ }^{5}$, \\ CHUNWU ZHANG ${ }^{6}$, LINHUA ZHANG ${ }^{7}$ and LI SUN $^{1}$ \\ ${ }^{1}$ Department of Rheumatology, The First Affiliated Hospital of Wenzhou Medical University, Wenzhou, Zhejiang 325003; \\ ${ }^{2}$ Zhejiang Center for Clinical Laboratory, Zhejiang Provincial People's Hospital, People's Hospital of \\ Hangzhou Medical College, Hangzhou, Zhejiang 310014; ${ }^{3}$ Division of Ophthalmic Genetics, Laboratory for Stem Cell \\ and Retinal Regeneration, Institute of Stem Cell Research, The Eye Hospital, Wenzhou Medical University, \\ Wenzhou, Zhejiang 325000; ${ }^{4}$ Department of Internal Medicine, Yueqing People's Hospital, Yueqing, Wenzhou, \\ Zhejiang 325600; ${ }^{5}$ Department of Nephrology, Wenzhou Central Hospital, Wenzhou, Zhejiang 325000; \\ ${ }^{6}$ Department of Injury Orthopaedics, The First Affiliated Hospital of Wenzhou University, Wenzhou, Zhejiang 325023; \\ ${ }^{7}$ Department of Clinical Laboratory, Yuhuan People's Hospital, Taizhou, Zhejiang 317600, P.R. China
}

Received August 2, 2019; Accepted January 8, 2020

DOI: $10.3892 /$ ijmm.2020.4501

\begin{abstract}
Gout is a common type of inflammatory arthritis that is clinically and genetically heterogeneous. The genetic aetiology remains unclear, and mainly relies on previous genome-wide association studies focused on sporadic cases. The present study aimed to identify the genetic basis of gout in three families using whole-exome sequencing (WES). WES was performed in the probands, and family members were involved in the co-segregation analysis. In total, three deleterious rare or novel missense mutations were identified in ATP-binding cassette super-family $\mathrm{G}$ member 2 ( $A B C G 2)$, protein kinase CGMP-dependent 2 (PRKG2) and adrenoceptor $\beta 3$ (ADRB3) genes in three different families. In addition, certain gout-associated candidate genes were revealed to be shared among the co-expression and protein-protein interaction (PPI) networks of $A B C G 2, P R K G 2$ and $A D R B 3$. Furthermore, the disease ontology analysis of the genes present in the co-expression network exhibited significant $(\mathrm{P}<0.05)$ enrichment in hyperuricemia, gout, cardiovascular system disease and metabolic
\end{abstract}

Correspondence to: Dr Linhua Zhang, Department of Clinical Laboratory, Yuhuan People's Hospital, 18 Changle Road, Taizhou, Zhejiang 317600, P.R. China

E-mail: zlhjy@126.com

Dr Li Sun, Department of Rheumatology, The First Affiliated Hospital of Wenzhou Medical University, Nanbaixiang, Ouhai, Wenzhou, Zhejiang 325003, P.R. China

E-mail: grassandsun@126.com

*Contributed equally

Key words: gout, whole-exome sequencing, large pedigrees, genetic variants, bioinformatics disease. In addition, genes involved in the PPI network were significantly enriched in the purine nucleoside monophosphate biosynthetic process, urate transport and biological processes associated with glycose metabolism. Collectively, to the best of our knowledge, the present study was the first to use WES to identify three candidate rare or novel deleterious mutations in three families with gout. The present results provided novel insights that may improve the current understanding of the molecular genetic basis underlying gout. Importantly, the present results may facilitate the improvement of clinical diagnosis and the development of novel personalized therapies.

\section{Introduction}

As a common type of inflammatory arthritis, gout is typically characterized by an elevated concentration of serum uric acid (hyperuricemia) and a subsequent deposition of monosodium urate (MSU) crystals in and around joints (1-3). The prevalence of gout in developed countries has been reported to be $1-3.9 \%$ (1-3). In addition, due to changes in dietary habits and an increasing aging population, the prevalence of gout has increased over past decades in China $(4,5)$. Patients with gout often present with metabolic diseases, including diabetes mellitus, hypertriglyceridemia and obesity, in addition to hypertension (6). In total, $16 \%$ of patients with gout exhibit ischemic heart disease and metabolic syndrome (7). Insulin resistance serves a key function in the pathogenesis of metabolic syndrome. Hyperuricemia in patients with metabolic syndrome is likely caused by insulin resistance, since insulin promotes uric acid and sodium reabsorption in the proximal tubule $(8,9)$.

Gout is etiologically heterogeneous as environmental and genetic components are involved (1-3). Although an unhealthy diet and lifestyle are risk factors for hyperuricemia, genetic factors were revealed to serve a greater function in the development of gout compared with environmental factors (10-12). 
Various previous genome-wide association studies (GWASs) have improved the understanding of the genes that regulate serum uric acid levels and increase susceptibility to gout (13-15). Among the loci revealed to be associated with gout, solute carrier family 2 member 9 and ATP-binding cassette super-family $\mathrm{G}$ member $2(A B C G 2)$ are the two most important genes identified to serve a function in gout development (10-12). Two important pathways, including renal and gut excretion of uric acid, have been revealed to regulate hyperuricemia levels, and each of these pathways are associated with glycolysis (16). However, previous studies based on GWAS research in independent individuals were only able to explain $\sim 7 \%$ of the variance in serum urate concentrations, and only a portion of those loci were confirmed to be associated with the risk of gout $(17,18)$.

Gout is heritable and has tendency to cluster in families $(12,19,20)$. In total, $20 \%$ of patients with gout were reported to have a family history of this disease. The risk of gout is significantly higher in patients who have first-degree relatives affected by this disease compared with the normal population (19). A previous segregation and linkage analysis of familial gout revealed an autosomal-arbitrary major gene model, which indicated a genetic basis for familial gout (20). A previous population-based study was performed in a Taiwanese population to estimate the degree of familial aggregation of gout, and it was revealed that genetic factors serve a substantial function in the development of gout (19).

The present study aimed to identify the potential pathogenic genetic causes of 3 pedigrees with a familial aggregation of gout using whole-exome sequencing (WES) technology and diverse bioinformatics analysis, including genetic interaction networks, disease ontology (DO) and gene ontology analysis.

\section{Materials and methods}

Patient recruitment. Patients $(\mathrm{n}=10)$ who were attending a clinic for arthritis were recruited between July 2016 and June 2017. Additionally, three families were recruited in the present study as: i) Presenting with autosomal dominant inheritance and ii) healthy family members agreed to participation in the present study. Patient information is summarised in Table I. The present study was conducted according to the principles outlined in the Declaration of Helsinki. The study protocol was approved by the Ethics Committee of The First Affiliated Hospital, Wenzhou Medical University (Wenzhou, China; approval no. 2018-020). Written informed consent was obtained from all participants or their guardians. Patients with gout were clinically evaluated by physicians based on the 2015 gout classification criteria by the American College of Rheumatology/European League Against Rheumatism Collaborative Initiative (21). Patients with inherited metabolic disorders, including Lesch-Nyhan syndrome, were excluded from the present study. Autoantibodies and human leukocyte antigen B27 (HLA-B27) tests were negative in all patients and X-ray analyses of the affected joints were performed to exclude other potential diseases. The classical symptoms of patients with gout are characterized by the rapid development of monoarticular arthritis, which is accompanied by swelling and redness of the first metatarsophalangeal joint (MTP1).
WES and variant calling. The human genome hg19 was used as the reference genome. Genomic DNA was extracted from peripheral blood leukocytes. In total, $2 \mu \mathrm{g}$ genomic DNA from each sample was sheared to fragment with a length of 150 base pairs (bp) using the Covaris S220. Subsequently, ligation of small fragments of DNA with A-tail and adapters was conducted. A genomic library was constructed subsequent to the amplification of adapter-ligated DNA using an Agilent SureSelect Library Prep kit (Agilent Technologies, Inc.) according to the manufacturer's protocol, and the samples from each individual were marked with a unique index. Whole-exome capture was performed using the Agilent SureSelect Human All Exon v5 kit (Agilent Technologies, Inc.) according to the manufacturer's protocol. High throughput sequencing was performed using an Illumina HiSeq 4000 sequencer (Illumina, Inc.).

All raw sequencing data obtained from these three families were processed in a similar manner, according to a customized bioinformatics pipeline (22). To remove sequence adapters and low-quality reads, the raw reads were filtered using the FastQC software program, version 1.11.4 (http://www.bioinformatics.babraham.ac.uk/projects/fastqc/). The filtration criteria excluded Phred-scaled quality scores $<30$ and read lengths $<80 \mathrm{bp}$. Subsequent to removing the low-quality reads, the remaining reads were used for further analysis. FastQ reads were aligned to the human reference genome (GRCh37/hg19) using the Burrows-Wheeler alignment tool (23) and further visualized using SplicingViewer software (24). Then, Genome Analysis ToolKit (GATK; version 4.0.10.0; https://gatk. broadinstitute.org/hc/en-us) was used to remove duplicated reads and reads mapped to multiple genome locations. In addition, local realignment and map quality score recalibration were performed. Candidate variants were then identified using the GATK Unified Genotype (version 4.0.10.0; https:/gatk. broadinstitute.org/hc/en-us).

Variant annotation and prioritization. mirTrios with an integrated ANNOVAR tool were used to annotate all the detected mutations according to an in-house pipeline (25). The minor allele frequency (MAF) was obtained for each variant from publicly available databases (25), including ExAC, UK10K, dbSNP147, 1000 Genomes and ESP6500, and from in-house exome data. The detected variants with a MAF $>0.01 \%$ present in any of the aforementioned databases were removed (26). Subsequently, the effects of the detected variants were predicted using four tools: i) Polymorphism Phenotyping v2 (PolyPhen2) (27); ii) Likelihood Ratio Test (LRT) (https://evomics.org/resources/likelihood-ratio-test/); iii) MutationTaster (28); and iv) Functional Analysis through Hidden Markov Models (FATHMM) (29). A missense variant was considered deleterious if the variant was predicted to be deleterious or damaging by $\geq 3$ of the four genetic prediction tools. The remaining variants were considered to be high-confidence causative variants.

Protein structural modelling. The amino acid sequence of human protein kinase CGMP-dependent 2 (PRKG2) was retrieved from Uniprot (http://www.uniprot. org/uniprot/Q13237). Crystal structures of the wild-type PRKG2 protein were obtained from the protein data bank 


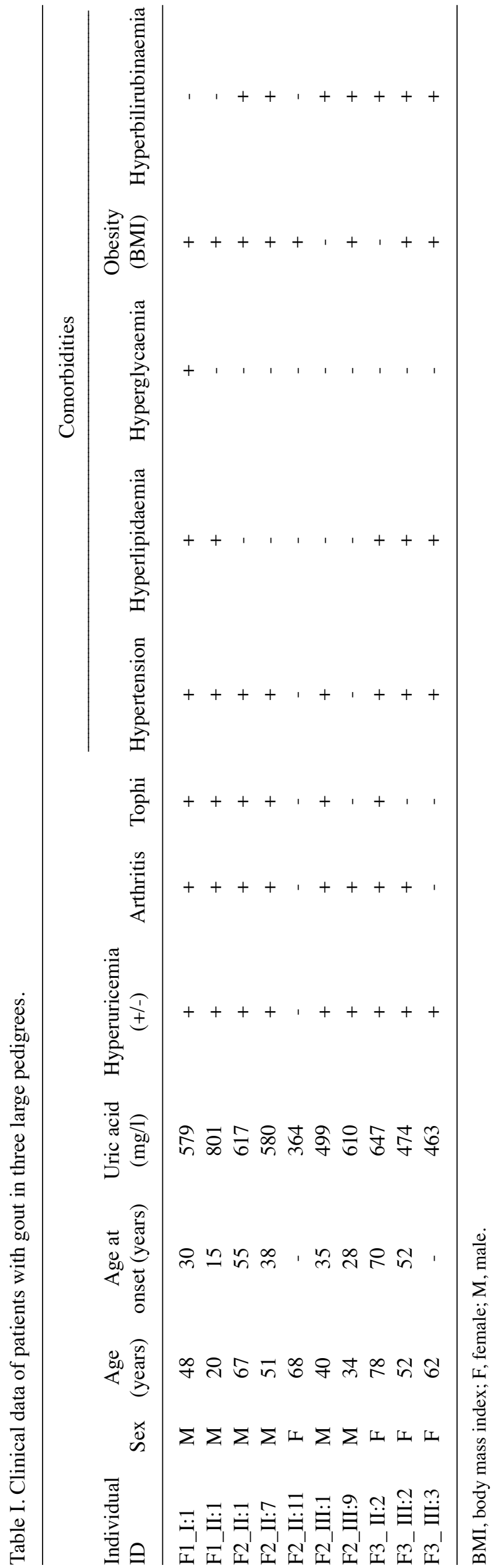

(PDB; http://www.rcsb.org/structure/5BV6) and visualized using PyMol software (version 1.8) (https://pymol.org/2/). Homology modelling of mutated PRKG2 protein structures was performed using SWISS-MODEL (https://swissmodel. expasy.org/) (30). In total, 120 residues were modelled in the PRKG2 structure, including 102 residues in the cGMP-binding region.

Collection of candidate genes for hyperuricemia and gout. Various candidate genes for hyperuricemia and gout were reported in previous studies. The literature search was performed in June 2017 by searching 'gout' AND 'genes' in PubMed (https://www.ncbi.nlm.nih.gov/pubmed). Then all genes in all literature obtained were included. Following the literature search, the candidate genes associated with hyperuricemia and gout were collected and are listed in Table SI.

Construction of gene co-expression and protein-protein interaction (PPI) networks. Temporally rich transcriptome data extracted from the Genotype-Tissue Expression (GTEx) project (https://gtexportal.org/home/, accessed January 2018) were used to build the co-expression network. The Pearson correlation coefficients ( $r$ ) for the gene co-expression levels were calculated for each pairwise combination between different genes. To investigate similarities among the genes forming the PPI network and analyse their functions, significantly enriched DO terms were identified using the $\mathrm{R}$ package DOSE (version 2.0) and a hypergeometric test (31). To assess function similarities between previously reported genes (Table SI) associated with gout and the core risk genes identified in the present study, a PPI network was built. PPI data downloaded from STRING V10 (https://string-db.org) (32) were used for PPI network analysis. To further investigate the gene functions in the PPI network, biological processes (BP) analysis was conducted using ClueGO v2.3.3 (33), a plug-in of Cytoscape.

\section{Results}

Characteristics of the patient cohort. The patient cohort for the present study was composed of three families (Figs. 1A, 2A and 3). All patients experienced acute monoarticular arthritis affecting the MTP1 and/or knee. The affected joint made walking difficult, was painful to the touch and was occasionally accompanied by fever. Tophus was observed in some of the patients (Fig. 1B), and metabolic disorders, including hypertension, diabetes and hyperlipemia, were identified. The clinical data are presented in Table I. The serum urate level was $>480 \mu \mathrm{mol} / 1$ in most cases, and in numerous cases it was $>600 \mu \mathrm{mol} / \mathrm{l}$. Synovial fluid from a number of the patients presented MSU crystals and erosions based on conventional radiography of the affected joint. Autoantibodies and HLA-B27 tests were negative in all patients (Table SII). All affected patients were diagnosed with gout.

Detection of candidate deleterious mutations. To determine the genetic etiology of these families, WES was performed in the three probands. In total, $\sim 13.53 \mathrm{~Gb}$ high-quality data was obtained on mean subsequent to removing sequencing 

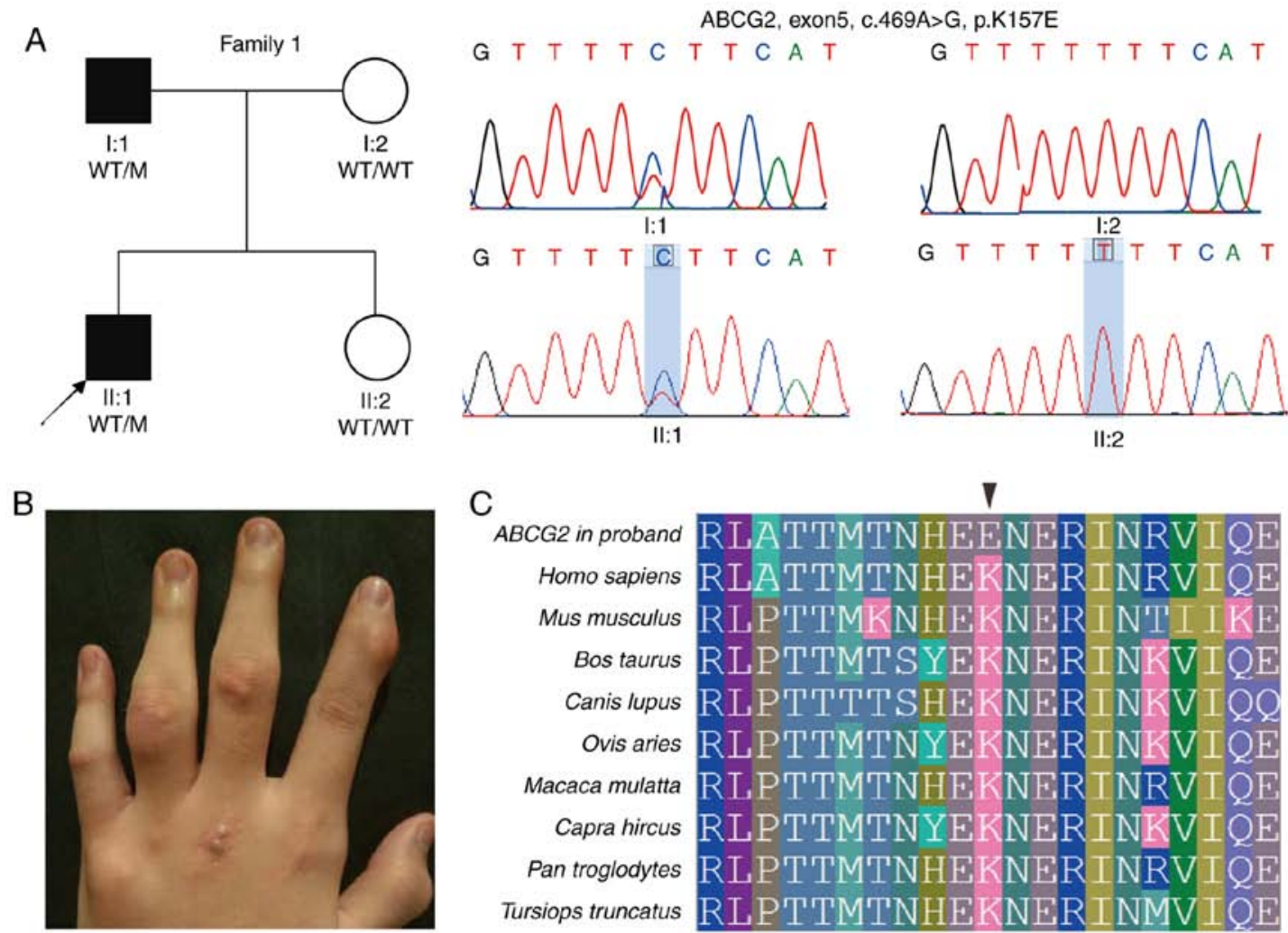

C ABCG2 in proband RLATTMTNHEENERINRVIQF Homo sapiens RLATTMTNHEKNERINRVIQE Mus musculus RLPTTMKNHEKNER INTI I KE Bos taurus RLPTTMTSYEKNERINKVIQE Canis lupus RLPTTTTSHEKNERINKVIQQ ovis aries RLPTTMTNYEKNERINKVIQE Macaca mulatta RI.PTTMTNHEKNERTNRVTQF

Capra hircus RLPTTMTNYEKNERINKVIQE

Pan troglodytes RLPTTMTNHEKNERINRVIQE Tursiops truncatus RLPTTMTNHEKNERINMVIOE

II:1
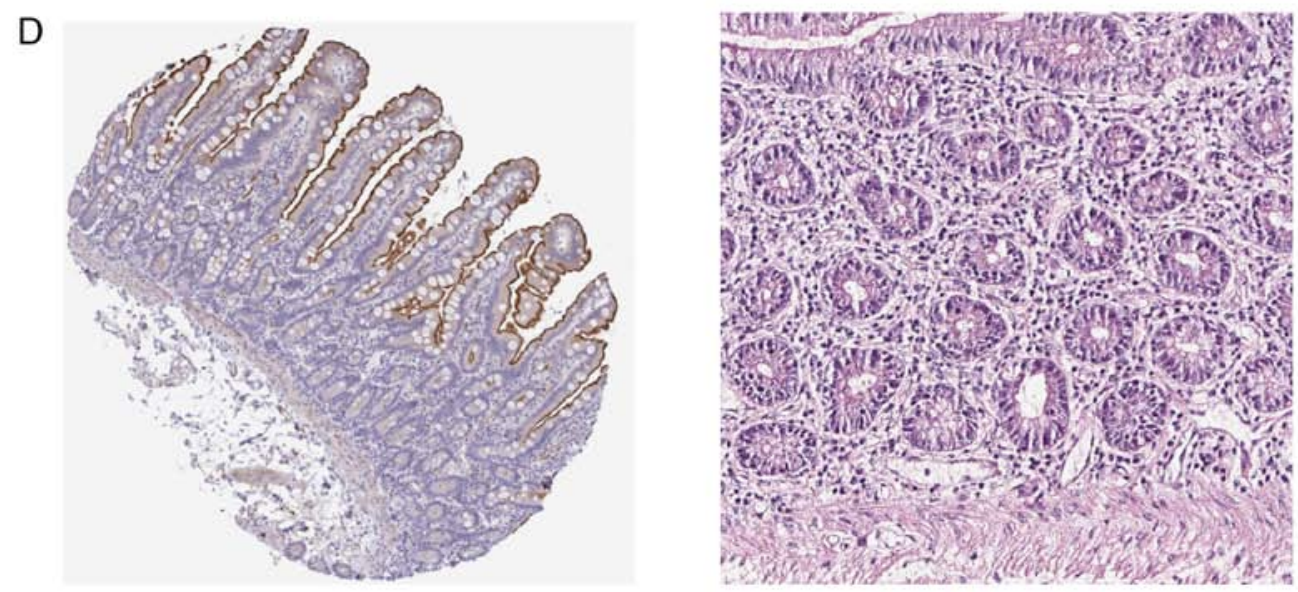

Figure 1. Identification of $A B C G 2$ mutation in Family 1. (A) Pedigree of Family 1. Sanger sequencing validation of the heterozygous missense mutation (c.469A > G, p.K157E). (B) Representative photographs of the proband reveals multiple urate deposits, indicating a severe disease burden. (C) Conservation of the $\mathrm{K} 157$ residue across different species revealing the conserved amino acid residues. (D) Luminal membrane expression of $A B C G 2$ in the intestine. Subset of cells in gastrointestinal tract exhibited a strong luminal membranous positivity. ABCG2, ATP-binding cassette super-family G member 2; WT, wild-type; M, mutant.

adapters and low-quality sequences (Table SIII). For each sample, $\geq 98.68 \%$ of the high-quality data was matched with the human reference genome $\mathrm{Hg} 19$. Effective sequence on target was $>4.91 \mathrm{~Gb}$, with a minimum of $39.10 \%$ fraction of effective bases on target following the removal of polymerase chain reaction duplications. The mean sequencing depth for each sample was 110.16 -fold, with $>99.00 \%$ of target regions being covered at a 4 -fold sequencing depth, $98.50 \%$ at 10 -fold depth and $97.20 \%$ at 20-fold depth. Collectively, the quality control data demonstrated a high reliability, which was fundamental for the subsequent analyses.

Subsequent to removing low-quality reads, adapters and duplicated reads from the raw sequencing data, a total of 541,954 single nucleotide variations (SNVs) and 84,415 indels were identified using the GATK tool, which included 67,707 SNVs and 2,200 indels in exonic and splicing regions. Subsequent to applying variant filtration against multiple databases, the number of rare SNVs and indels causing protein change with MAF $<0.001$ was reduced to 228 and 29, respectively. As a result, following effect prediction by Polyphen2, LRT, Mutation Taster and FATHMM, the variants predicted to be deleterious by $>2$ prediction tools were validated by Sanger sequencing. Finally, three SNVs in the coding regions of three gout-associated genes were retained and confirmed following Sanger sequencing validation (Table II). 
Sanger sequencing validation and co-segregation testing. Analysis of WES data indicated a missense mutation (p.Lys157Glu) in the $A B C G 2$ gene in the proband F1:II:1 of family 1 presenting typical symptoms of gout (Fig. 1 and Table I). The ABCG2 protein is involved in the excretion of urate from the intestine and kidney, and its dysfunction causes extrarenal urate underexcretion type (34) and/or renal urate underexcretion type gout (35). The heterozygous missense mutation (c.469A $>\mathrm{G}$ ) causing a lysine (Lys) to glutamic acid (Glu) substitution was located at the amino acid 157. Subsequently, Sanger sequencing confirmed the presence of this mutation in the patient's affected father while his unaffected mother and sister did not present the $A$ to $G$ change at cDNA nucleotide 469 (Fig. 1A). The MAF of p.Lys157Glu was $8.24 \times 10^{-6}$ and was predicted to be deleterious by all four effect prediction tools (Table II). Additionally, the residue 157Lys is highly conserved among different vertebrate species (Fig. 1C). Subsequently, the tissue specificity of the expression of the human $A B C G 2$ was examined in all major tissues and organs using the Human Protein Atlas database (https://www. proteinatlas.org). $A B C G 2$ exhibited the most abundant protein expression in the small intestine (Fig. 1D). The results reflected abundant mRNA expression in the luminal membrane of the intestine with 136.8 transcripts per million (TPM).

Another heterozygous missense mutation (c.752A $>\mathrm{G}$; p.Asn251Ser) located in exon 5 of $P R K G 2$ gene was detected in proband F2:II:1 and his affected brother F2:II:9, as well as in the patient III9 (Fig. 2A). cGMP-dependent protein kinase 2 (cGKII)/PRKG2 is involved in the regulation of aldosterone and renin secretion (36). In total, nine unaffected family members presented wild-type alleles (Fig. 2A). Importantly, this mutation was not previously observed in any public database. The residue 251Asn was revealed to be evolutionarily conserved (Fig. 2B) and PRKG2 expression was identified to be enriched in the small intestine (18.36 TPM), mainly in glandular cells (Fig. 2C). In the F3, the proband F3:II:1 and his two affected sons shared the same missense mutation consisting of a $\mathrm{G}$ to $\mathrm{C}$ substitution (c.12G $>\mathrm{C}$ ) in $A D R B 3$ (Fig. 3). $A D R B 3$ is part of the adrenergic system, which is involved in the regulation of lipid metabolism and glucose homeostasis (37). This single-nucleotide change resulted in a non-synonymous substitution (p.Trp4Cys). PolyPhen2 predicted that this variant was likely damaging (Table II). Sanger sequencing validated that six healthy family members presented homozygous wild-type alleles for ADRB3 (Fig. 3). Similarly to the aforementioned two residues, this position was predicted to be evolutionary conserved by the GERP++ tool (https://omictools.com/gerp-tool).

Protein structural modelling. Previous GWAS studies identified single nucleotide polymorphisms (SNPs) in these three genes that were associated with gout (38-40). All SNPs and three candidate mutations identified in the present study were mapped to schematic representations of $A B C G 2, P R K G 2$ and $A D R B 2$ genes (Fig. 4A). Except for the two mutations in $P R K G 2$, all the other mutations were located in the protein functional domains. Furthermore, p.Lys157Glu mutation in $A B C G 2$ was located in the same highly conserved $A B C$ transporter domain as the pathogenic missense variant Gln141Lys, which has been revealed to be associated with an increase in 
A
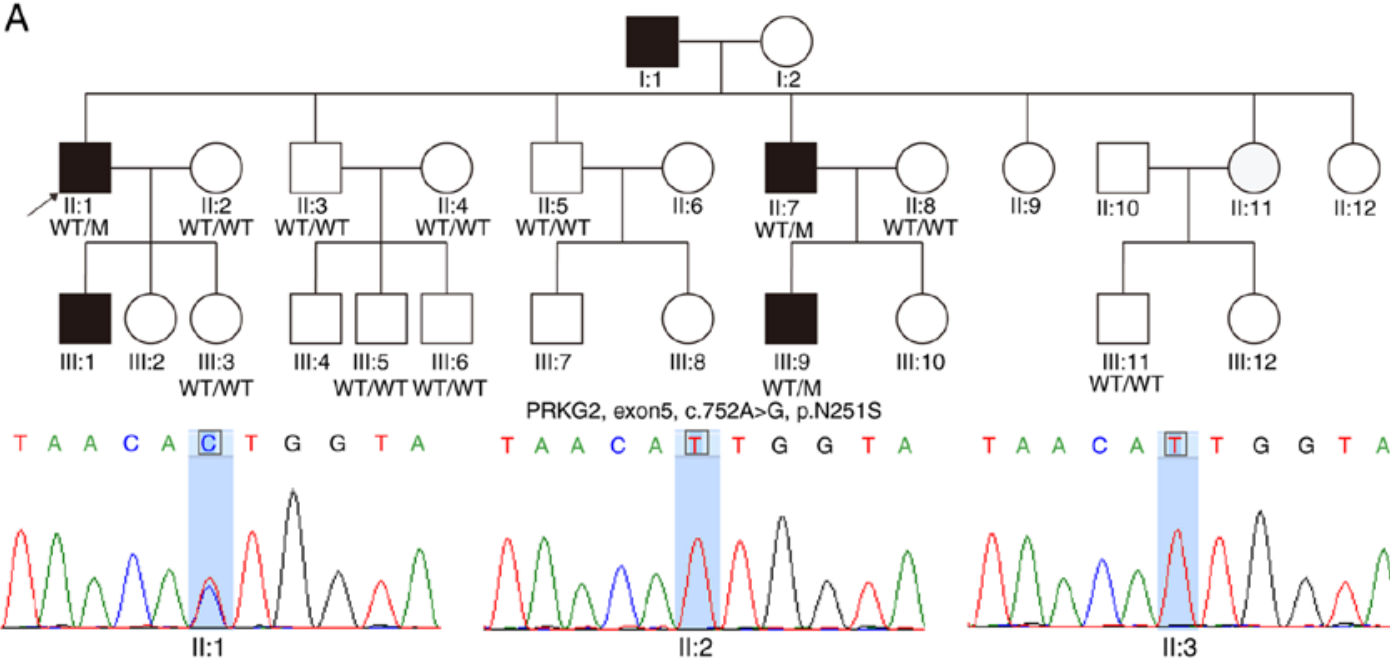

PRKG2, exon5, c.752A>G, p.N251s
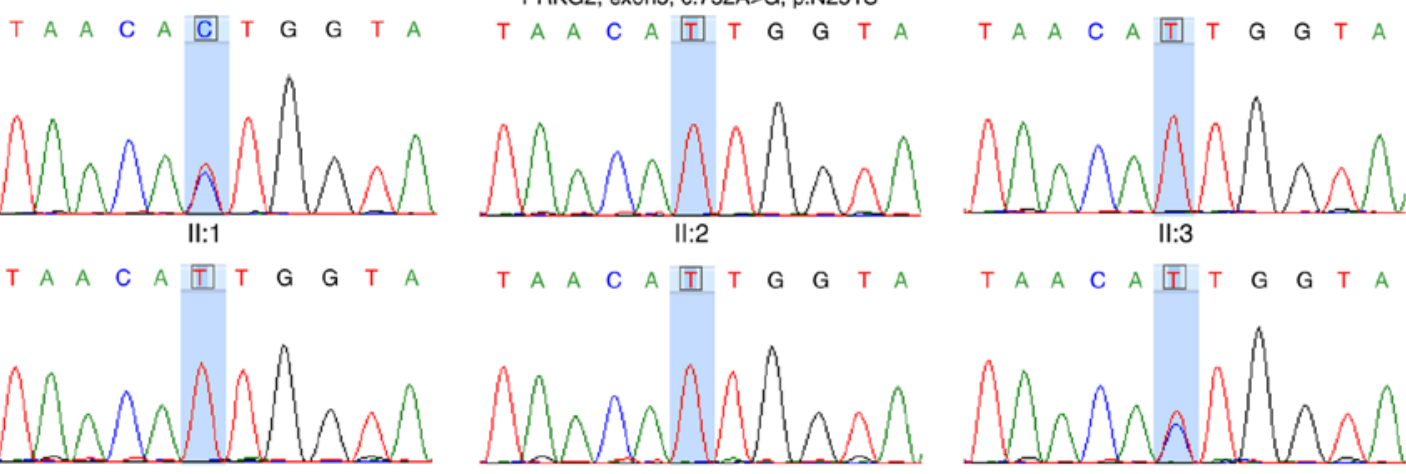

II:4
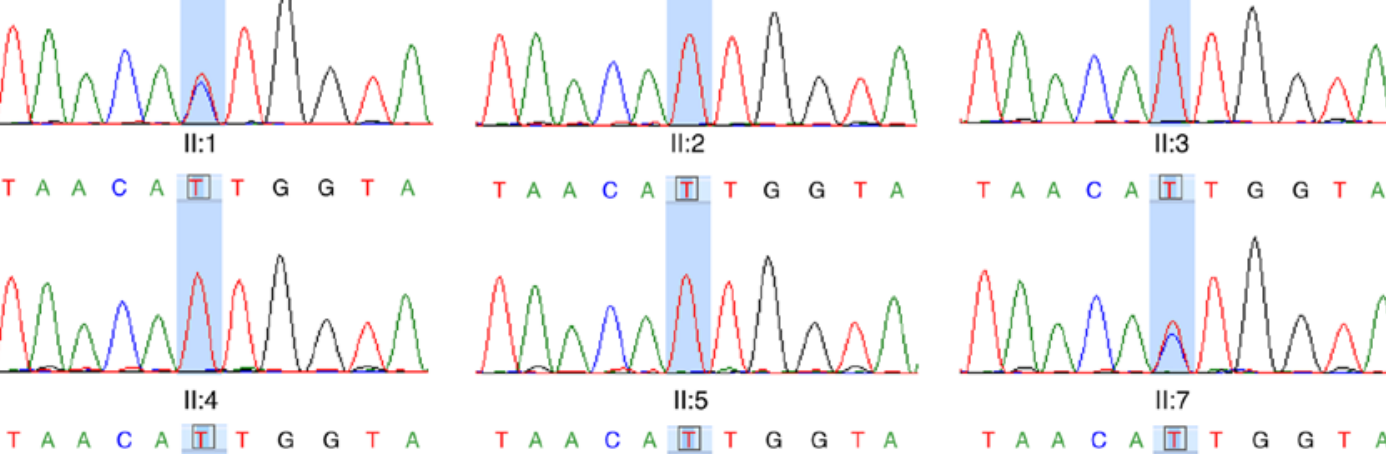

II:5

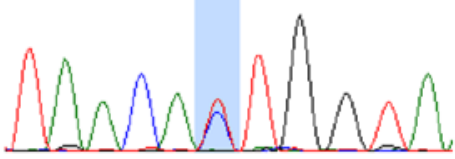

II:7
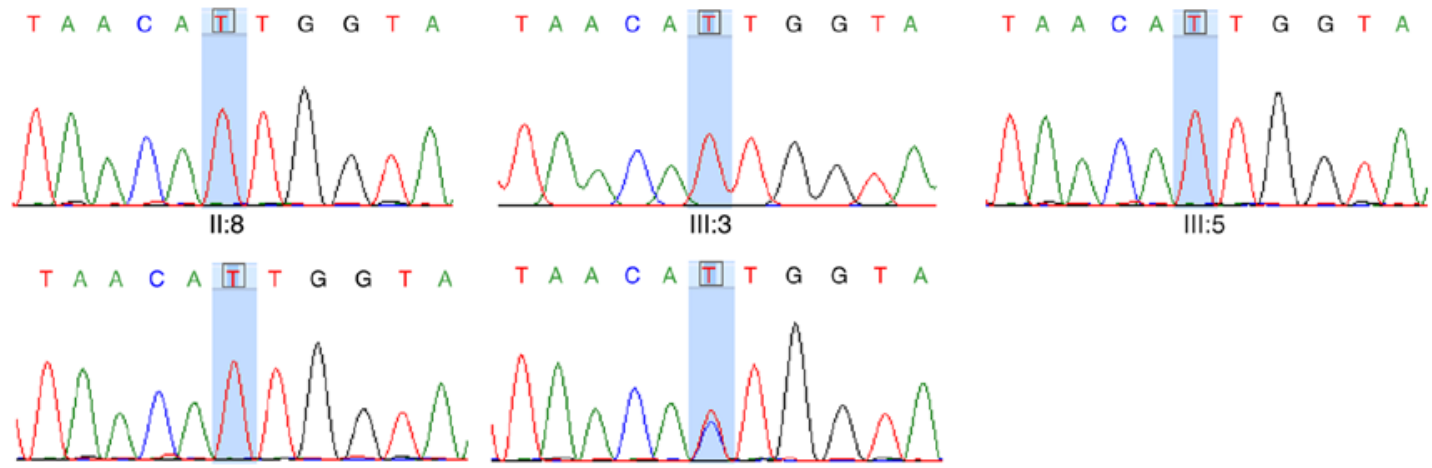

III:6

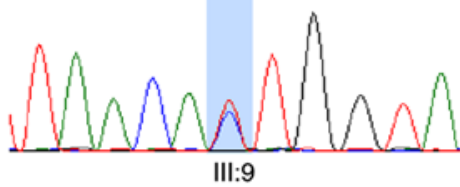

$\checkmark$
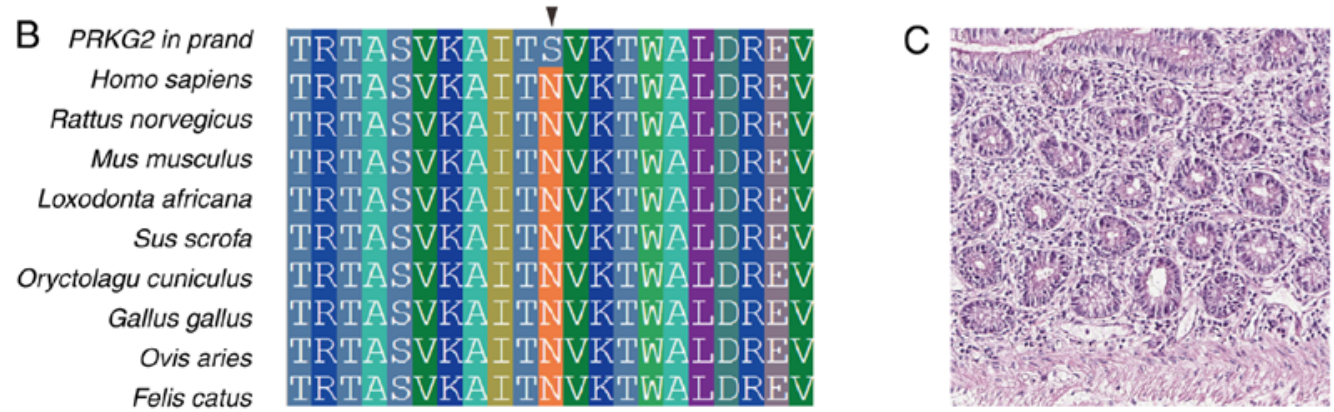

Figure 2. Identification of PRKG2 mutation in Family 2. (A) Pedigree of Family 2. Sanger sequencing confirmed the heterozygous missense mutation (c.A752G). (B) Conservation of the variant c.752A $>\mathrm{G}$ among different species. (C) In situ hybridization revealing PRKG2 expression in the human small intestine. PRKG2, protein kinase CGMP-dependent 2; WT, wild-type; M, mutant.

serum uric acid levels (41). The Asn251Ser mutation was in the cGMP-binding region of PRKG2; however, rs768867227 and rs10033237 SNPs, which were previously reported to cause gout, were present in non-coding regions (42).

In order to further study the functional defects caused by mutations in the protein structure, the potential structural differences between the wild-type and mutant proteins were investigated. Therefore, numerous differences were identified between the wild-type and mutant $P R K G 2$ proteins. The wild-type structure (5BV6) was downloaded from the PDB.
In the wide-type protein, there were seven hydrogen bonds at residue 251 (Asn); one bond was revealed between Asn251 and Glu191, and the other six connected Asn251 to water molecules (Fig. 4B). The mutated structural modelling revealed the formation of two different hydrogen bonds, one between the mutated Ser251 and Thr250, and another between the mutated Ser251 and Tyr189 (Fig. 4B).

Functional analysis of the co-expression and PPI networks. Previous studies have identified that gout-associated genes 


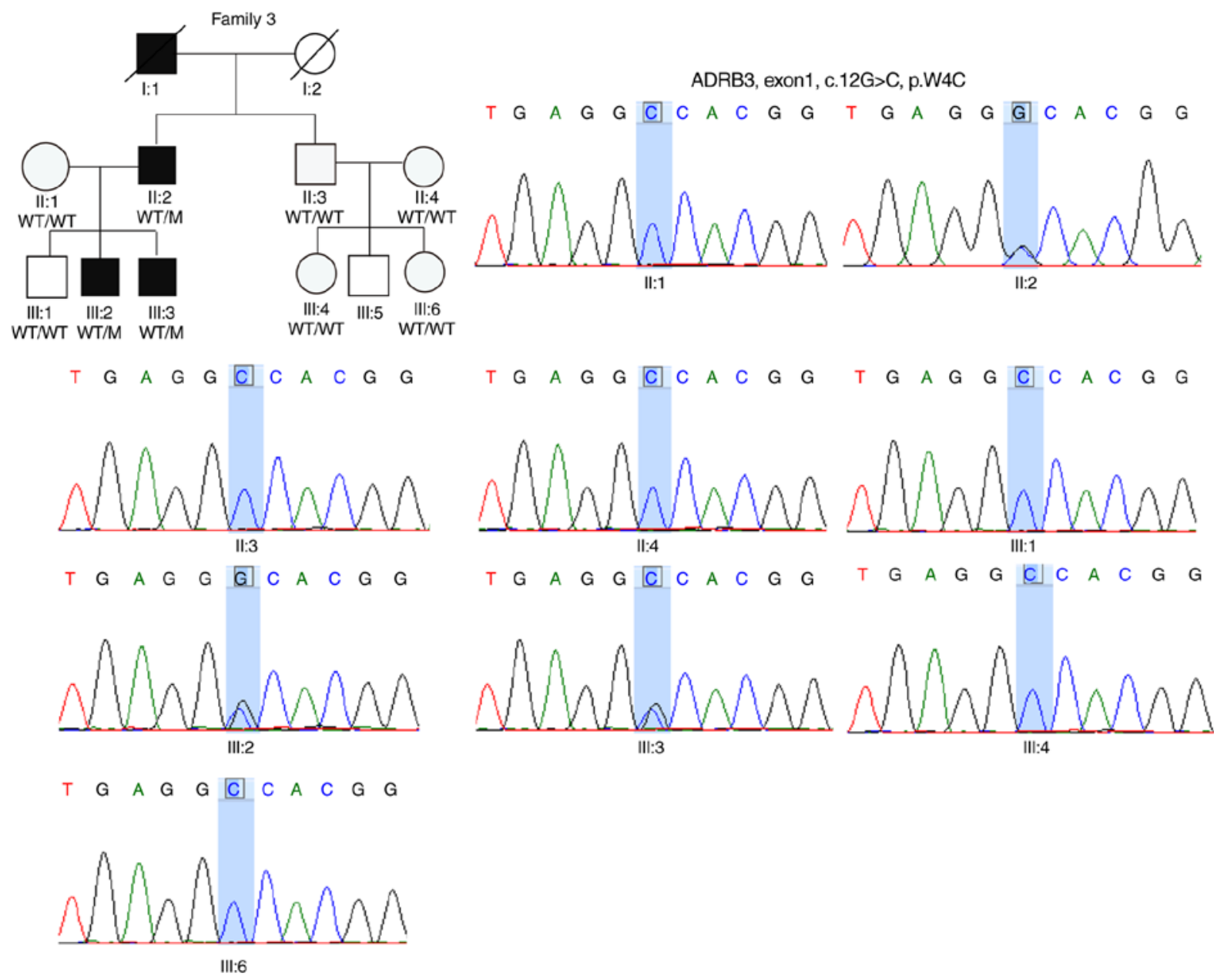

Figure 3. Identification of $A D R B 3$ mutation in Family 3. Pedigree of Family 3. Sanger sequencing confirmed the heterozygous missense mutation (c.A752G). ADRB3, adrenoceptor $\beta 3$; WT, wild-type; $M$, mutant.

are closely associated with urate excretion (10-12). To further investigate the expression pattern of the three candidate genes ( $A B C G 2, P R K G 2$ and $A D R B 2$ ) identified in the present study and to examine whether their expression patterns were similar to other gout-associated genes identified in previous studies, the transcriptomic data in the small intestinal tissue from the GTEx project were analysed. Based on the co-expression network, these three genes demonstrated similar expression patterns with numerous gout-associated genes, with a Pearson correlation coefficient ranging between 0.602 and 0.898 (Fig. 5A). Among these three genes, the number of candidate genes co-expressed with the $A B C G 2$ gene was the largest $(\mathrm{n} \leq 39)$. Furthermore, to identify functional similarities among these co-expressed genes that may have a function in the development of gout, significantly enriched DO terms were identified using the $\mathrm{R}$ package DOSE. Among the top ten most statistically significant DO terms with Bonferroni corrected $\mathrm{P}<0.05$ (Fig. 5B; Table SIV), the majority of the terms were associated with cardiovascular and metabolic diseases, supporting the idea that gout is often accompanied by metabolic syndrome. The two directly associated terms, hyperuricemia [false discovery rate $(\mathrm{FDR})=8.02 \times 10^{-14}$, hypergeometric test $]$ and gout $\left(\mathrm{FDR}=2.32 \times 10^{-12}\right.$, hypergeometric test) were the most statistically significant, indicating the significant enrichment of the co-expressed genes in hyperuricemia and gout.
To investigate the association between the three candidate genes and other gout candidate genes, data from the STRING v10 database were analysed. The STRING database contains PPIs, including physical and functional associations. As a result, a total of 79 candidate genes were included in the PPI network (Fig. 6A) with the interaction score ranging between 150 and 967. Solute carrier family 22 member 1 exhibited the strongest interaction with $A B C G 2$, which is expressed in the kidney and mediates the transport of xenobiotics, endogenous organic anions and urate (43). PRKG2 exhibited the strongest interaction with inositol 1,4,5-trisphosphate receptor type 1, which was previously reported to be associated with high serum uric acid concentrations (44). In the next step, all 79 candidate genes in the PPI network were used in the BP with ClueGO plugin. A total of 40 enriched BP terms were divided into eight groups (Fig. 6B) and terms in the same group had similar biological functions. In total, two groups served direct functions in the metabolism of uric acid, and the terms in the two groups with the most significant statistical significance were 'purine nucleoside monophosphate biosynthetic process' and 'urate transport'. 'Glycometabolism' and 'anion transport' groups were also identified following data enrichment analysis. The detailed results of the BP analysis are presented in Table SV. 
A

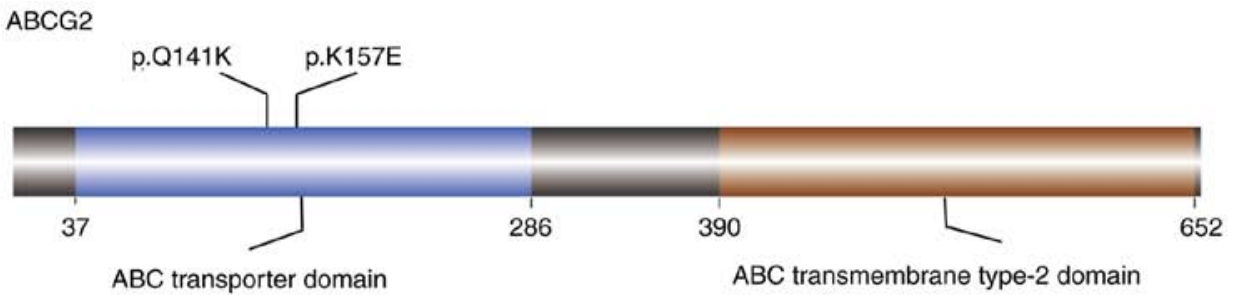

ADRB3
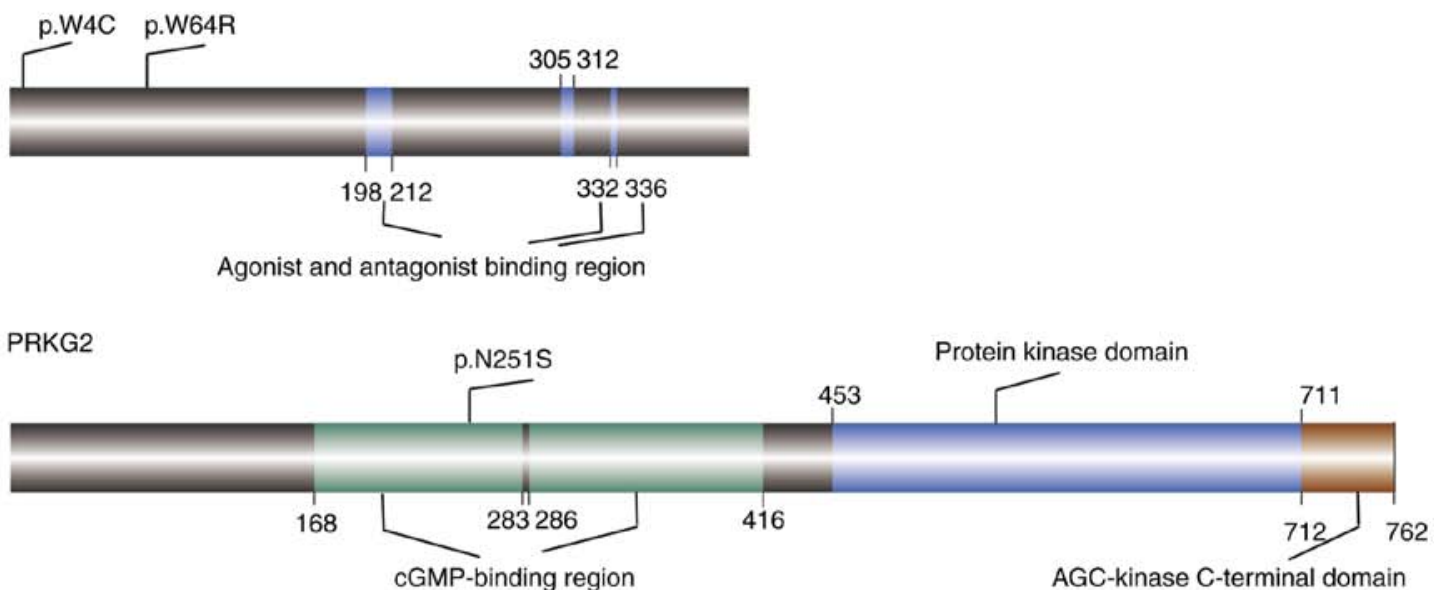

B

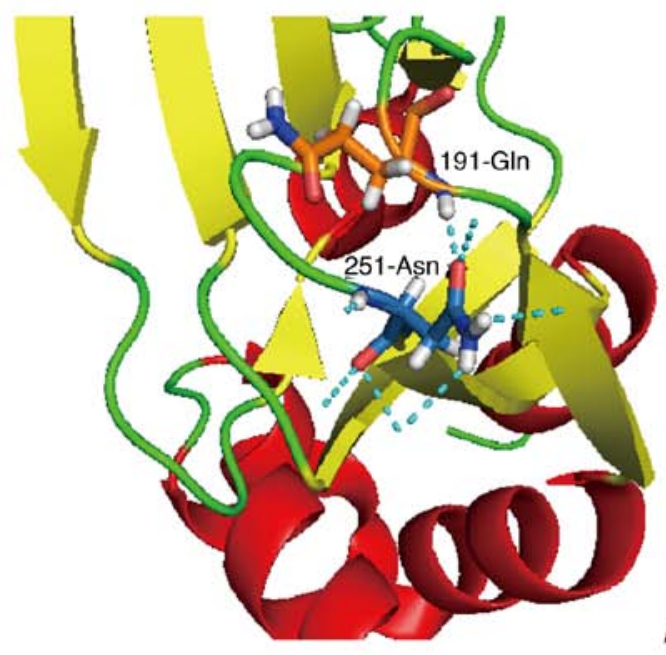

Wild-type PRKG protein

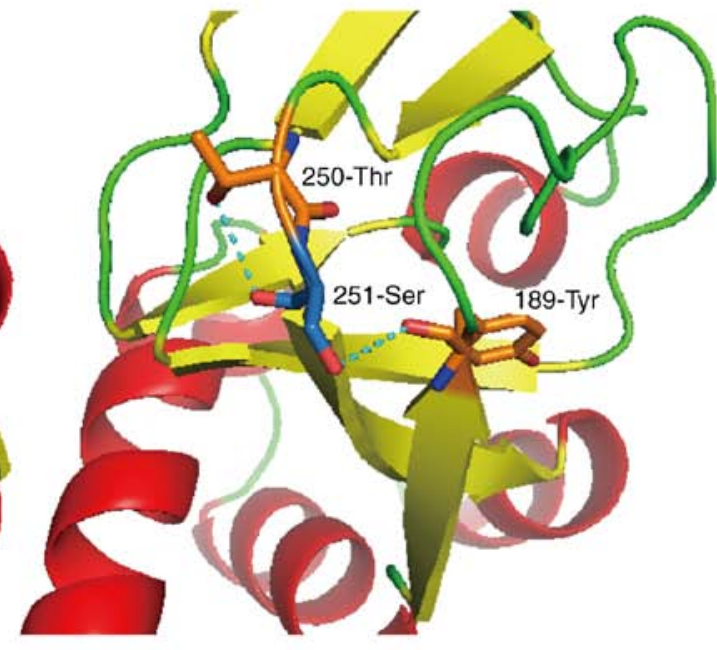

Mutant PRKG2 protein

Figure 4. Modelling structure analysis of mutations identified. (A) Schematic map of the $A B C G 2, A D R B 3$ and $P R K G 2$ genes and their domains with the sites of the variants identified in the present study and previous studies associated with gout. (B) Crystal structures of wild-type $P R K G 2$ protein and the mutated structural modeling of PRKG2 protein. ABCG2, ATP-binding cassette super-family G member 2; PRKG2, protein kinase CGMP-dependent 2; ADRB3, adrenoceptor $\beta 3$.

\section{Discussion}

Gout is a complex disorder characterised by clinical and genetic heterogeneity, and the genetic mechanism underlying gout remains unclear, mainly because i) previous studies focused on sporadic cases; and ii) genotyping chips were unable to identify rare or novel variants. To address these two critical issues, the present study aimed to reveal candidate rare/novel mutations in large pedigrees with gout aggregation. A previous complex segregation and linkage analysis of familial gout revealed an autosomal-arbitrary major gene model (20). WES is able to provide insights into the pathogeny of hereditary diseases and extend molecular diagnosis (45-48). Therefore, WES was performed in three families with gout. Subsequent to employing previously established filtering strategies, three candidate variants were identified in these three families.

In the proband F1:II:1, one novel missense mutation was revealed in $A B C G 2$ (c.469A $>\mathrm{G}$, p.Lys157Glu), and it was predicted to be deleterious by all four functional prediction tools. $A B C G 2$ has been reported to be an important factor involved in the reduction of urate transport rates (40) and in a pathway regulating fructose metabolism, which is associated with obesity $(13,49)$. The patient in F1 presenting the $A B C G 2$ mutation exhibited early onset-gout and was overweight, and 

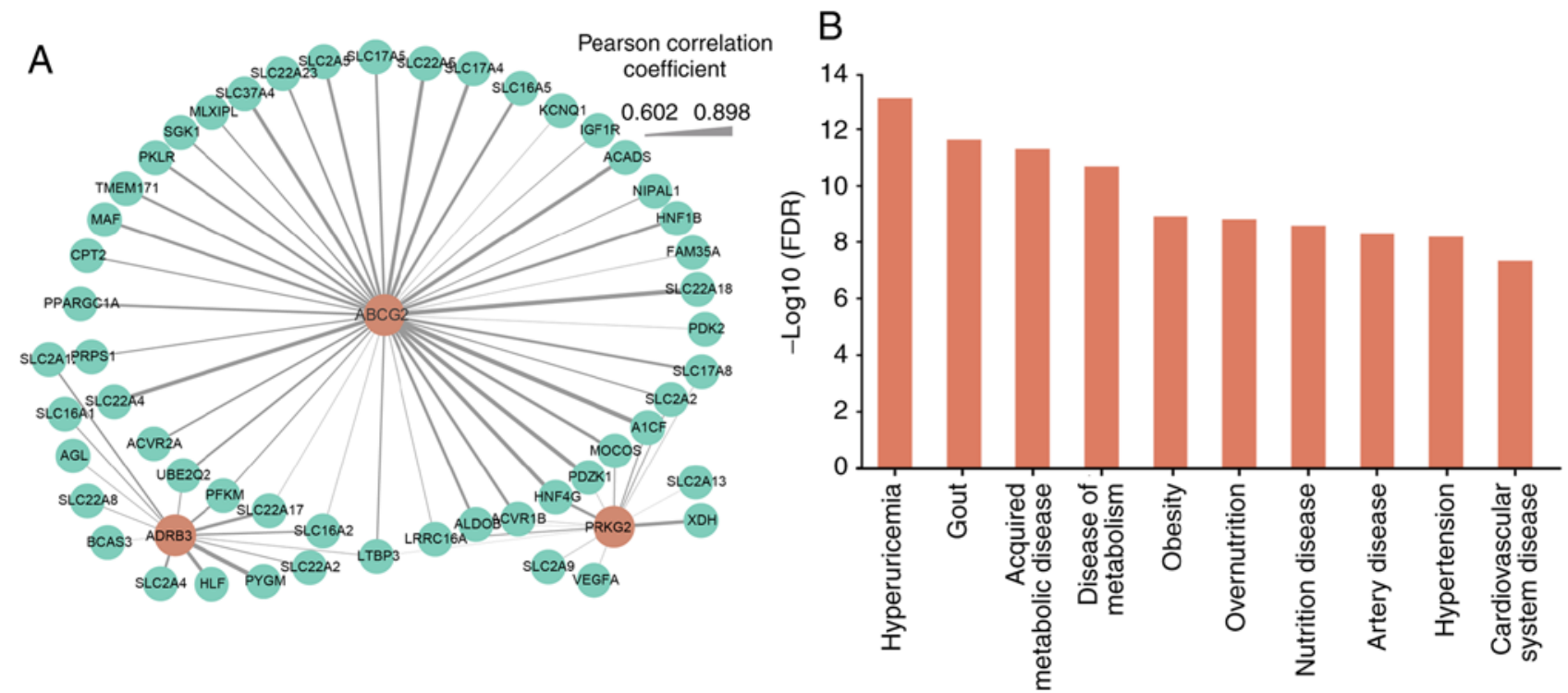

Figure 5. Co-expression network analysis. (A) Co-expression network analysis of $A B C G 2, P R K G 2$ and $A D R B 3$ with other gout candidate genes. Gene co-expression levels were estimated using the Pearson correlation coefficients (r) between each pair of genes. (B) Disease Ontology enrichment analysis of genes in the co-expression network. ABCG2, ATP-binding cassette super-family G member 2; PRKG2, protein kinase CGMP-dependent 2; ADRB3, adrenoceptor $\beta 3$.

A

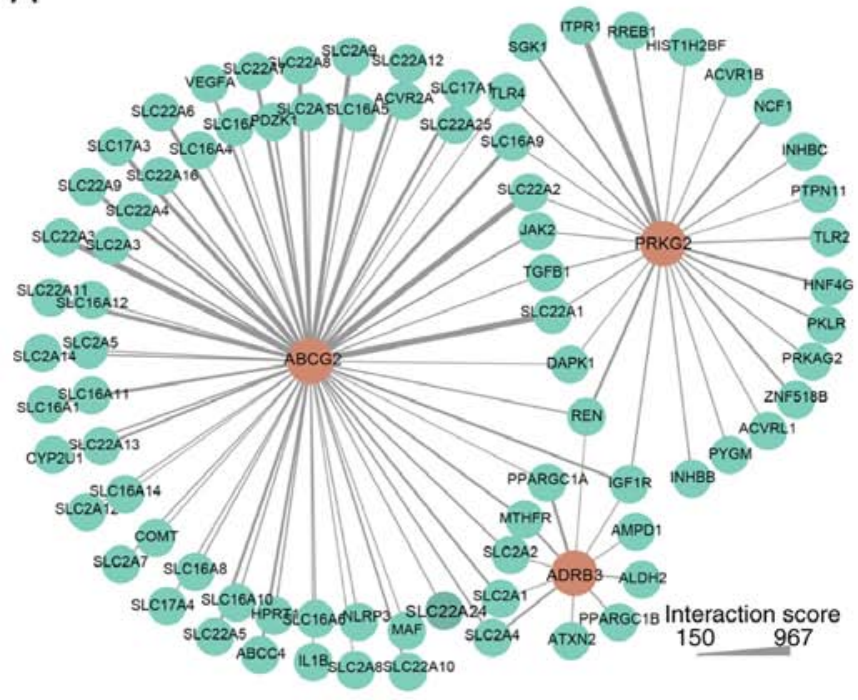

B

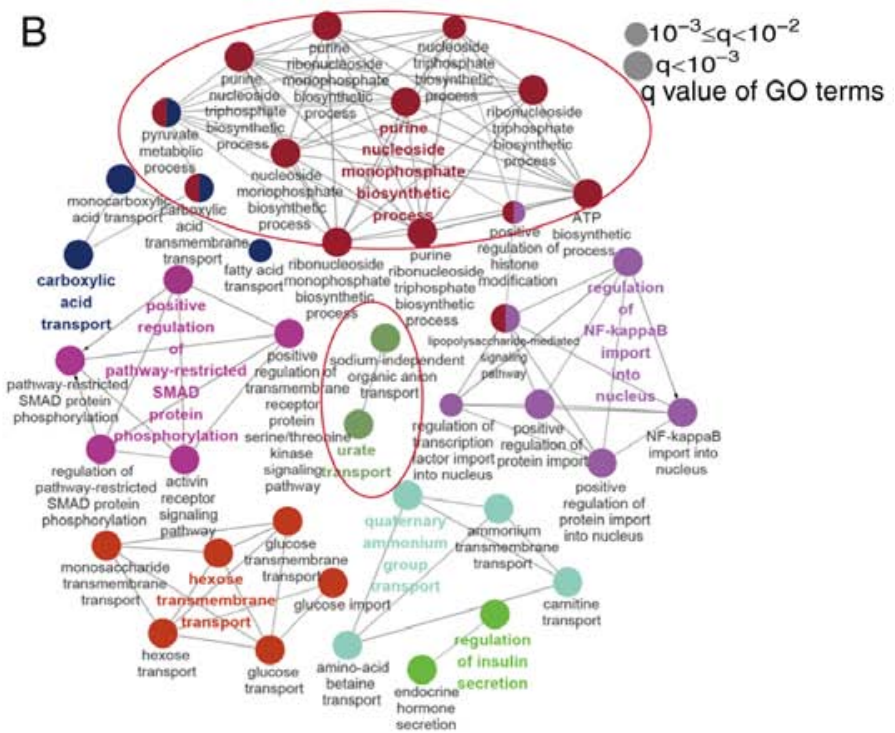

Figure 6. Genetic interaction network analysis. (A) Genetic interaction network analysis of ABCG2, PRKG2 and ADRB3. Nodes denote genes, and edges denote interactions between two genes. Interaction score represents the degree of genetic interaction. (B) Biological process analysis of genes in genetic interaction network utilizing the ClueGO plug-in. ABCG2, ATP-binding cassette super-family G member 2; PRKG2, protein kinase CGMP-dependent 2; ADRB3, adrenoceptor $\beta 3$.

these symptoms are in line with the pathological features of gout. The two most commonly reported dysfunctional SNPs are Gln126Ter (rs72552713) and Gln141LysK (rs2231142), which are located in the ABC domain, which is considered to be critical for the interactions between the intracellular loops of the transmembrane portion of the protein (40). The presence of the $\mathrm{Q} 141 \mathrm{~K}$ polymorphism in the $\mathrm{ABC}$ transporter domain was previously reported to induce a 2 -fold decrease in urate efflux (50). The mutation p.Lys157Glu identified in the present study is located in the ABC transporter domain, indicating that the pathogenic mechanism of this mutation is caused by dysfunctions in this functional domain. Furthermore, a previous study has identified three common and 19 rare non-synonymous variants of $A B C G 2$ in patients with gout and functional assays were performed to determine the urate transport activity of each $A B C G 2$ variant (51). Almost all rare variants identified in the present study exhibited lower urate transport and almost completely inhibited $A B C G 2$ activity compared with the wild-type protein, as assessed by functional assays performed to determine the urate transport activity of each $A B C G 2$ variant.

The second mutation identified was present in $A D R B 3$, which encodes a $\beta$-3-adrenergic receptor and has been revealed to serve an important function in the regulation of lipolysis and glucose homeostasis $(37,39)$. Hyperuricemia and gout are 
closely associated with metabolic disorders, including obesity, dyslipidaemia, glucose intolerance and hypertension $(52,53)$. Wang et al (20) and Huang et al (54) reported that a p.Trp64Arg variant was associated with hyperuricemia in Chinese male patients. A similar study investigating Spanish patients identified that a p.Trp64Arg polymorphism in ADRB3 gene may increase the risk of hyperuricemia (55). Therefore, the missense mutation c. $12 \mathrm{G}>\mathrm{C}$ in the $A D R B 3$ gene revealed in the present study was spatially near the same intracellular loop affected in the p.Trp64Arg mutation, and it is thus expected to be involved in the development of basal metabolic diseases including dyslipidaemia, which was confirmed by the fact that patients with gout in Family 3 had hyperlipemia and hyperbilirubinemia.

The third mutation identified in the present study was located in the PRKG2 gene. Accumulating evidence has demonstrated that the cGMP signalling pathway serves important functions in urate cycles, and PRKG2 is a cGKII gene (42). One case-control study revealed that there was a correlation between the polymorphisms rs768867227 and rs10033237 in PRKG2, and gout susceptibility (56). Each of these polymorphisms are located in non-coding regions. Although the biological function of combined rs10033237 and rs7688672 in the PRKG2 gene has not been elucidated, dysfunctions in PRKG2 may result in hypertension by destroying the renin-angiotensin-aldosterone system, and may result in hyperuricemia (42). The renin-angiotensin-aldosterone system in the patients in Family 3 carrying heterozygous missense mutations (c.752A $>\mathrm{G}$ and Asn251Ser) in PRKG2 was potentially impaired. An impaired renin-angiotensin-aldosterone system may cause hyperuricemia, which was consistent with the presence of hypertension in the patients in Family 3. Furthermore, a previous study (57) demonstrated that PRKG2 serves a key function in mediating M1 polarization and phagocytotic activity by regulating the levels of monosodium urate and lipopolysaccharides.

Excretion of uric acid requires specialized transporters located in renal tubule cells and intestinal epithelial cells (58). Accordingly, direct intestinal secretion is considered as a substantial contributor to the extra-renal elimination of uric acid (59). It is estimated that $30 \%$ of uric acid is excreted by the intestine (60). Numerous gout-associated genes are highly expressed in the small intestine, including $A B C G 2$ and $P R K G 2$ detected in the present study (10-12). The co-expression network revealed that gout-associated genes share similar expression patterns with $A B C G 2, P R K G 2$ and $A D R B 3$ in the small intestine. $A B C G 2$ is a well-characterized urate transporter in the intestine, and the molecular function of $A B C G 2$ was previously identified (10-12). The functional interactions between proteins are crucial for their biological function, and their systematic characterization may increase current understanding of molecular systems biology (61). The STING network constructed using $A B C G 2, P R K G 2$ and $A D R B 3$ contained a number of gout-associated genes, suggesting that the pathogenic mechanism of these genes may share the same pathway.

Gout is a metabolic disorder caused by urate overproduction and/or reduced urate excretion (62). According to previous studies, gout is associated with five major metabolic syndromes and/or consequences of metabolic syndrome: Hypertension, cardiovascular disease, insulin resistance and diabetes, obesity and hyperlipidaemia (63-65). In the present study, a DO analysis was performed on the genes identified in the co-expression network, and three DO terms associated with the cardiovascular system, including cardiovascular system disease, hypertension and artery disease, were in the top 10 significantly enriched terms. By directly impairing the vascular endothelium and the renal system, high serum urate levels may increase blood pressure (66). Other enriched terms were mainly associated with obesity. Following a data analysis performed on data from 517 participants in the Bogalusa Heart Study, Muntner et al (67) suggested that an elevated body mass index was associated with high levels of uric acid. Furthermore, the BP analysis of the genes in the PPI network contained terms including 'hexose transmembrane transport' and 'regulation of insulin secretion', and the two groups involved in glycose metabolism have been reported to affect the transport of uric acid (68-70). The majority of the enriched BP terms were revealed to be involved in metabolite transports, which are closely associated with urate transport.

Despite recent advancements in the understanding of the mechanism underlying gout, the number of families involved in the present study was small; therefore, the statistical power for the autosomal dominant inheritance model in gout was limited, although the model was previously proposed according to statistical analyses based on large sample sizes $(19,20)$. In addition, no functional genomics studies were performed in the present study. Therefore, the sample size of the study should be expanded to provide stronger evidence for the Mendelian genetic inheritance of gout. Copy number variations were not analysed in the present study $(71,72)$. Furthermore, functional experiments are necessary to further determine the function of the mutations detected in gout pathogenesis.

Collectively, to the best of our knowledge, the present study is the first to use WES to dissect rare or novel genetic variants using pedigree and family aggregation analyses. Among the three families, three deleterious mutations in three different gout pathogenic genes were identified. The present results increase the current knowledge of the genotypic heterogeneity underlying the phenotypic heterogeneity of gout, which assists not only clinical diagnoses but also potential personalized therapy.

\section{Acknowledgements}

Not applicable.

\section{Funding}

The present study was supported by the Zhejiang Provincial Natural Science Foundation (grant no. LY20H100001) and the Wenzhou Science and Technology Bureau (grant no. Y20180129).

\section{Availability of data and materials}

All the datasets generated and/or analysed in the present study are available from the corresponding author upon reasonable request.

\section{Authors' contributions}

$\mathrm{XX}$ and $\mathrm{JJ}$ conceived and designed the study. $\mathrm{ZC}$ and $\mathrm{ZZ}$ performed the experiments. $\mathrm{HC}$ and $\mathrm{CZ}$ collected and analysed 
the experimental data. LZ wrote the article. LS helped revise the article. All authors read and approved the final manuscript.

\section{Ethics approval and consent to participate}

This study was conducted according to the principles outlined in the Declaration of Helsinki. The study protocol was approved by the Ethics Committee of The First Affiliated Hospital, Wenzhou Medical University (Wenzhou, China; approval no. 2018-020). Written informed consent was obtained from all participants or their guardians.

\section{Patient consent for publication}

The patients provided written informed consent regarding the publication of the case details and any associated images.

\section{Competing interests}

The authors declare that they have no competing interests.

\section{References}

1. Bardin T and Richette P: The epidemiology and genetic of gout. Presse Med 40: 830-835, 2011 (In French).

2. Kuo CF, Grainge MJ, Zhang W and Doherty M: Global epidemiology of gout: Prevalence, incidence and risk factors. Nat Rev Rheumatol 11: 649-662, 2015.

3. Zhu Y, Pandya BJ and Choi HK: Prevalence of gout and hyperuricemia in the US general population: The national health and nutrition examination survey 2007-2008. Arthritis Rheum 63 3136-3141, 2011.

4. Liu R, Han C, Wu D, Xia X, Gu J, Guan H, Shan Z and Teng W: Prevalence of hyperuricemia and gout in mainland China from 2000 to 2014: A systematic review and meta-analysis. Biomed Res Int 2015: 762820, 2015

5. Lu X, Li X, Zhao Y, Zheng Z, Guan S and Chan P: Contemporary epidemiology of gout and hyperuricemia in community elderly in Beijing. Int J Rheum Dis 17: 400-407, 2014.

6. Yu TF, Dorph DJ and Smith H: Hyperlipidemia in primary gout. Semin Arthritis Rheum 7: 233-244, 1978.

7. Vázquez-Mellado J, García CG, Vázquez SG, Medrano G, Ornelas M, Alcocer L, Marquez A and Burgos-Vargas R: Metabolic syndrome and ischemic heart disease in gout. J Clin Rheumatol 10: 105-109, 2004.

8. Facchini F, Chen YD, Hollenbeck CB and Reaven GM: Relationship between resistance to insulin-mediated glucose uptake, urinary uric acid clearance, and plasma uric acid concentration. JAMA 266: 3008-3011, 1991.

9. Kaplan NM: The deadly quartet. Upper-body obesity, glucose intolerance, hypertriglyceridemia, and hypertension. Arch Intern Med 149: 1514-1520, 1989.

10. Choi HK, Zhu Y and Mount DB: Genetics of gout. Curr Opin Rheumatol 22: 144-151, 2010.

11. Mikuls TR and Saag KG: New insights into gout epidemiology. Curr Opin Rheumatol 18: 199-203, 2006.

12. Riches PL, Wright AF and Ralston SH: Recent insights into the pathogenesis of hyperuricaemia and gout. Hum Mol Genet 18 R177-R184, 2009.

13. Dehghan A, Kottgen A, Yang Q, Hwang SJ, Kao WL, Rivadeneira F, Boerwinkle E, Levy D, Hofman A, Astor BC, et al: Association of three genetic loci with uric acid concentration and risk of gout: A genome-wide association study. Lancet 372: 1953-1961, 2008.

14. Kolz M, Johnson T, Sanna S, Teumer A, Vitart V, Perola M, Mangino M, Albrecht E, Wallace C, Farrall M, et al: Meta-analysis of 28,141 individuals identifies common variants within five new loci that influence uric acid concentrations. PLoS Genet 5: e1000504, 2009.

15. Yang Q, Kottgen A, Dehghan A, Smith AV, Glazer NL, Chen MH, Chasman DI, Aspelund T, Eiriksdottir G, Harris TB, et al: Multiple genetic loci influence serum urate levels and their relationship with gout and cardiovascular disease risk factors. Circ Cardiovasc Genet 3: 523-530, 2010.
16. Merriman TR: An update on the genetic architecture of hyperuricemia and gout. Arthritis Res Ther 17: 98, 2015.

17. Kottgen A, Albrecht E, Teumer A, Vitart V, Krumsiek J, Hundertmark C, Pistis G, Ruggiero D, O'Seaghdha CM, Haller T, et al: Genome-wide association analyses identify 18 new loci associated with serum urate concentrations. Nat Genet 45 : 145-154, 2013.

18. Li C, Li Z, Liu S, Wang C, Han L, Cui L, Zhou J, Zou H, Liu Z, Chen J, et al: Genome-wide association analysis identifies three new risk loci for gout arthritis in Han Chinese. Nat Commun 6: $7041,2015$.

19. Kuo CF, Grainge MJ, See LC, Yu KH, Luo SF, Valdes AM, Zhang $\mathrm{W}$ and Doherty M: Familial aggregation of gout and relative genetic and environmental contributions: A nationwide population study in Taiwan. Ann Rheum Dis 74: 369-374, 2015.

20. Wang WH, Chang SJ, Wang TN, Cheng LS, Feng YP, Chen CJ, Huang $\mathrm{CH}$ and Ko YC: Complex segregation and linkage analysis of familial gout in Taiwanese aborigines. Arthritis Rheum 50: 242-246, 2004

21. Neogi T, Jansen TL, Dalbeth N, Fransen J, Schumacher HR, Berendsen D, Brown M, Choi H, Edwards NL, Janssens HJ, et al: 2015 Gout classification criteria: An American college of rheumatology/European league against rheumatism collaborative initiative. Arthritis Rheumatol 67: 2557-2568, 2015.

22. Wang T, Liu Q, Li X, Wang X, Li J, Zhu X, Sun ZS and Wu J: RRBS-analyser: A comprehensive web server for reduced representation bisulfite sequencing data analysis. Hum Mutat 34: 1606-1610, 2013.

23. Li H and Durbin R: Fast and accurate long-read alignment with Burrows-Wheeler transform. Bioinformatics 26: 589-595, 2010.

24. Liu Q, Chen C, Shen E, Zhao F, Sun Z and Wu J: Detection, annotation and visualization of alternative splicing from RNA-Seq data with SplicingViewer. Genomics 99: 178-182, 2012.

25. Li J, Jiang Y, Wang T, Chen H, Xie Q, Shao Q, Ran X, Xia K, Sun ZS and Wu J: mirTrios: An integrated pipeline for detection of de novo and rare inherited mutations from trios-based next-generation sequencing. J Med Genet 52: 275-281, 2015.

26. Huang XF, Huang F, Wu KC, Wu J, Chen J, Pang CP, Lu F, Qu J and Jin ZB: Genotype-phenotype correlation and mutation spectrum in a large cohort of patients with inherited retinal dystrophy revealed by next-generation sequencing. Genet Med 17: 271-278, 2015.

27. Adzhubei IA, Schmidt S, Peshkin L, Ramensky VE, Gerasimova A, Bork P, Kondrashov AS and Sunyaev SR: A method and server for predicting damaging missense mutations. Nat Methods 7: 248-249, 2010.

28. Schwarz JM, Cooper DN, Schuelke M and Seelow D: MutationTaster2: Mutation prediction for the deep-sequencing age. Nat Methods 11: 361-362, 2014.

29. Shihab HA, Gough J, Mort M, Cooper DN, Day IN and Gaunt TR: Ranking non-synonymous single nucleotide polymorphisms based on disease concepts. Hum Genomics 8: 11, 2014.

30. Waterhouse A, Bertoni M, Bienert S, Studer G, Tauriello G, Gumienny R, Heer FT, de Beer TAP, Rempfer C, Bordoli L, et al: SWISS-MODEL: Homology modelling of protein structures and complexes. Nucleic Acids Res 46: W296-W303, 2018.

31. Yu G, Wang LG, Yan GR and He QY: DOSE: An R/Bioconductor package for disease ontology semantic and enrichment analysis. Bioinformatics 31: 608-609, 2015.

32. Szklarczyk D, Gable AL,LyonD,Junge A, Wyder S,Huerta-Cepas J, Simonovic M, Doncheva NT, Morris JH, Bork P, et al: STRING v11: Protein-protein association networks with increased coverage, supporting functional discovery in genome-wide experimental datasets. Nucleic Acids Res 47: D607-D613, 2019.

33. Bindea G, Mlecnik B, Hackl H, Charoentong P, Tosolini M, Kirilovsky A, Fridman WH, Pagès F, Trajanoski Z and Galon J: ClueGO: A Cytoscape plug-in to decipher functionally grouped gene ontology and pathway annotation networks. Bioinformatics 25: 1091-1093, 2009.

34. Ichida K, Matsuo H, Takada T, Nakayama A, Murakami K, Shimizu T, Yamanashi Y, Kasuga H, Nakashima H, Nakamura T, et al: Decreased extra-renal urate excretion is a common cause of hyperuricemia. Nat Commun 3: 764, 2012.

35. Matsuo H, Nakayama A, Sakiyama M, Chiba T, Shimizu S, Kawamura Y, Nakashima H, Nakamura T, Takada Y, Oikawa Y, et al: ABCG2 dysfunction causes hyperuricemia due to both renal urate underexcretion and renal urate overload. Sci Rep 4: $3755,2014$.

36. Vaandrager AB, Hogema BM and de Jonge HR: Molecular properties and biological functions of cGMP-dependent protein kinase II. Front Biosci 10: 2150-2164, 2005. 
37. Krief S, Lönnqvist F, Raimbault S, Baude B, Van Spronsen A Arner P, Strosberg AD, Ricquier D and Emorine LJ: Tissue distribution of beta 3-adrenergic receptor mRNA in man. J Clin Invest 91: 344-349, 1993

38. Sakiyama M, Matsuo H, Chiba T, Nakayama A, Nakamura T, Shimizu S, Morita E, Fukuda N, Nakashima H, Sakurai Y, et al Common variants of cGKII/PRKG2 are not associated with gout susceptibility. J Rheumatol 41: 1395-1397, 2014.

39. Wang B, Meng D, Wang J, Jia Z, Zhoub S, Liu S, Chu N, Han L, Zhang K, Ma X and Li C: Positive correlation between Beta-3-Adrenergic Receptor (ADRB3) gene and gout in a Chinese male population. J Rheumatol 38: 738-740, 2011.

40. Woodward OM, Köttgen A, Coresh J, Boerwinkle E, Guggino WB and Köttgen M: Identification of a urate transporter, ABCG2, with a common functional polymorphism causing gout. Proc Natl Acad Sci USA 106: 10338-10342, 2009.

41. Woodward OM, Tukaye DN, Cui J, Greenwell P, Constantoulakis LM, Parker BS, Rao A, Köttgen M, Maloney PC and Guggino WB: Gout-causing Q141K mutation in ABCG2 leads to instability of the nucleotide-binding domain and can be corrected with small molecules. Proc Natl Acad Sci USA 110 5223-5228, 2013.

42. Guo M, Cheng Z, Li C, Li S, Li M, Wang M, Xu J, Tang Y, Wang Y, Qiu W and Liu X: Polymorphism of rs7688672 and rs10033237 in cGKII/PRKG2 and gout susceptibility of Han population in northern China. Gene 562: 50-54, 2015.

43. Sakiyama M, Matsuo H, Shimizu S, Nakashima H, Nakayama A, Chiba T, Naito M, Takada T, Suzuki H, Hamajima N, et al: A common variant of organic anion transporter4(OAT4/SLC22A11) gene is associated with renal underexcretion type gout. Drug Metab Pharmacokinet 29: 208-210, 2014.

44. Chittoor G, Kent JW Jr, Almeida M, Puppala S, Farook VS, Cole SA, Haack K, Göring HH, MacCluer JW, Curran JE, et al: GWAS and transcriptional analysis prioritize ITPR1 and CNTN4 for a serum uric acid 3p26 QTL in Mexican Americans. BMC Genomics 17: 276, 2016.

45. Bergmann C: Advances in renal genetic diagnosis. Cell Tissue Res 369: 93-104, 2017.

46. Wu J, Shen E, Shi D, Sun Z and Cai T: Identification of a novel Cys146X mutation of SOD1 in familial amyotrophic lateral sclerosis by whole-exome sequencing. Genet Med 14: 823-826, 2012

47. Huang XF, Xiang L, Cheng W, Cheng FF, He KW, Zhang BW, Zheng SS, Han RY, Zheng YH, Xu XT, et al: Mutation of IPO13 causes recessive ocular coloboma, microphthalmia, and cataract. Exp Mol Med 50: 53, 2018

48. Huang XF, Xiang L, Fang XL, Liu WQ, Zhuang YY, Chen ZJ, Shen RJ, Cheng W, Han RY, Zheng SS, et al: Functional characterization of CEP250 variant identified in nonsyndromic retinitis pigmentosa. Hum Mutat 40: 1039-1045, 2019.

49. Dalbeth N, House ME, Gamble GD, Pool B, Horne A, Purvis L, Stewart A, Merriman M, Cadzow M, Phipps-Green A and Merriman TR: Influence of the ABCG2 gout risk $141 \mathrm{~K}$ allele on urate metabolism during a fructose challenge. Arthritis Res Ther 16: R34, 2014.

50. Basseville A and Bates SE: Gout, genetics and $A B C$ transporters F1000 Biol Rep 3: 23, 2011.

51. Higashino T, Takada T, Nakaoka H, Toyoda Y, Stiburkova B, Miyata H, Ikebuchi Y, Nakashima H, Shimizu S, Kawaguchi M, et al: Multiple common and rare variants of ABCG2 cause gout. RMD Open 3: e000464, 2017.

52. Choi HK and Ford ES: Prevalence of the metabolic syndrome in individuals with hyperuricemia. Am J Med 120: 442-447, 2007.

53. Choi HK, Ford ES, Li C and Curhan G: Prevalence of the metabolic syndrome in patients with gout: The third national health and nutrition examination survey. Arthritis Rheum 57: 109-115, 2007.

54. Huang Q, Zhang LF, Cheng Y, Zhao YC, Si L, Gao Y and Wei W: Trp64Arg (rs4994) polymorphism of $\beta 3$-adrenergic receptor gene is associated with hyperuricemia in a Chinese male population. Clin Chem Lab Med 51: 1755-1760, 2013.
55. Morcillo S, Rojo-Martinez G, Martin-Nunez GM, GómezZumaquero JM, García-Fuentes E, Ruiz de Adana M, de la Cruz Almaraz M and Soriguer F: Trp64Arg polymorphism of the ADRB3 gene predicts hyperuricemia risk in a population from southern Spain. J Rheumatol 37: 417-421, 2010.

56. Chang SJ, Tsai MH, Ko YC, Tsai PC, Chen CJ and Lai HM: The cyclic GMP-dependent protein kinase II gene associates with gout disease: Identified by genome-wide analysis and case-control study. Ann Rheum Dis 68: 1213-1219, 2009.

57. Liao WT, You HL, Li C, Chang JG, Chang SJ and Chen CJ: Cyclic GMP-dependent protein kinase II is necessary for macrophage M1 polarization and phagocytosis via toll-like receptor 2. J Mol Med (Berl) 93: 523-533, 2015.

58. Xu X, Li C, Zhou P and Jiang T: Uric acid transporters hiding in the intestine. Pharm Biol 54: 3151-3155, 2016

59. Hosomi A, Nakanishi T, Fujita T and Tamai I: Extra-renal elimination of uric acid via intestinal efflux transporter BCRP/ABCG2. PLoS One 7: e30456, 2012.

60. So A and Thorens B: Uric acid transport and disease. J Clin Invest 120: 1791-1799, 2010.

61. Szklarczyk D, Franceschini A, Kuhn M, Simonovic M, Roth A, Minguez P, Doerks T, Stark M, Muller J, Bork P, et al: The STRING database in 2011: Functional interaction networks of proteins, globally integrated and scored. Nucleic Acids Res 39: D561-D568, 2011.

62. Yamanaka H: Gout and hyperuricemia in young people. Curr Opin Rheumatol 23: 156-160, 2011.

63. Billiet L, Doaty S, Katz JD and Velasquez MT: Review of hyperuricemia as new marker for metabolic syndrome. ISRN Rheumatol 2014: 852954, 2014.

64. Puig JG and Martinez MA: Hyperuricemia, gout and the metabolic syndrome. Curr Opin Rheumatol 20: 187-191, 2008.

65. Thottam GE, Krasnokutsky S and Pillinger MH: Gout and metabolic syndrome: A tangled web. Curr Rheumatol Rep 19: $60,2017$.

66. Kang DH, Han L, Ouyang X, Kahn AM, Kanellis J, Li P, Feng L, Nakagawa T, Watanabe S, Hosoyamada M, et al: Uric acid causes vascular smooth muscle cell proliferation by entering cells via a functional urate transporter. Am J Nephrol 25: 425-433, 2005.

67. Muntner P, Srinivasan S, Menke A, Patel DA, Chen W and Berenson G: Impact of childhood metabolic syndrome components on the risk of elevated uric acid in adulthood: The Bogalusa Heart Study. Am J Med Sci 335: 332-337, 2008.

68. Fam AG: Gout, diet, and the insulin resistance syndrome. J Rheumatol 29: 1350-1355, 2002.

69. Gheita TA, El-Fishawy HS, Nasrallah MM and Hussein H: Insulin resistance and metabolic syndrome in primary gout: Relation to punched-out erosions. Int J Rheum Dis 15: 521-525, 2012.

70. Perez-Ruiz F, Aniel-Quiroga MA, Herrero-Beites AM, Chinchilla SP, Erauskin GG and Merriman T: Renal clearance of uric acid is linked to insulin resistance and lower excretion of sodium in gout patients. Rheumatol Int 35: 1519-1524, 2015.

71. Huang XF, Mao JY, Huang ZQ, Rao FQ, Cheng FF, Li FF, Wang QF and Jin ZB: Genome-wide detection of copy number variations in unsolved inherited retinal disease. Invest Ophthalmol vis Sci 58: 424-429, 2017.

72. Huang XF, Wu J, Lv JN, Zhang X and Jin ZB: Identification of false-negative mutations missed by next-generation sequencing in retinitis pigmentosa patients: A complementary approach to clinical genetic diagnostic testing. Genet Med 17: 307-311, 2015.

This work is licensed under a Creative Commons Attribution-NonCommercial-NoDerivatives 4.0 International (CC BY-NC-ND 4.0) License. 